Check for updates

Cite this: RSC Adv., 2017, 7, 52391

Received 8th September 2017 Accepted 2nd November 2017

DOI: 10.1039/c7ra10013b rsc.li/rsc-advances

\section{Microwave-assisted reduction method under nitrogen atmosphere for synthesis and electrical conductivity improvement of reduced graphene oxide (rGO) $\uparrow$}

\begin{abstract}
Ferry Iskandar, (DD *ab Utiya Hikmah, ${ }^{a}$ Erythrina Stavila ${ }^{\mathrm{b}}$ and Akfiny H. Aimon ${ }^{a}$
A facile method to synthesize $\mathrm{rGO}$ using a microwave-assisted method under $\mathrm{N}_{2}$-atmosphere conditions is reported. Two different reducing agents were used in this research, hydrazine hydrate and L-ascorbic acid (L-AA). The reduction of graphene oxide was completed in 3 minutes by the microwave-assisted method under $\mathrm{N}_{2}$-atmosphere conditions. The structures and morphologies of both rGOs prepared by this new method were confirmed by attenuated total reflectance-Fourier transform infrared (ATR-FTIR) spectrometry, X-ray diffraction (XRD), Raman spectroscopy, scanning electron microscopy (SEM), transmission electron microscopy (TEM) and energy dispersive $X$-ray spectrometry (EDX), confirming the formation of rGO. Four-point probe measurements showed an electrical conductivity of $18.10 \mathrm{~S} \mathrm{~cm}^{-1}$ for the $\mathrm{rGO}$ reduced by hydrazine hydrate and $12.02 \mathrm{~S} \mathrm{~cm}^{-1}$ for the $\mathrm{rGO}$ reduced by L-AA. Combining this new method with annealing at $300{ }^{\circ} \mathrm{C}$ for 30 min resulted in a higher electrical conductivity: up to $23.26 \mathrm{~S} \mathrm{~cm}^{-1}$ for the $\mathrm{rGO}$ reduced by hydrazine hydrate and up to $16.19 \mathrm{~S} \mathrm{~cm}^{-1}$ for the rGO reduced by L-AA. This study provides a new perspective on synthesizing $\mathrm{rGO}$ with high electrical conductivity using an effective and efficient method.
\end{abstract}

\section{Introduction}

Carbon-based materials are extensively used in technological development, chemical industries, marine and railroad transportation, etc. ${ }^{1,2}$ Interest in the field of nanoscience and nanofabrication research is increasing rapidly. This is shown by the vast use of carbon-based nanostructured materials, which include carbon nanotubes, ${ }^{2,3}$ fullerenes, ${ }^{2,3}$ nanodiamonds, ${ }^{3}$ graphene quantum dots, ${ }^{4}$ reduced graphene oxide $(\mathrm{rGO}),{ }^{5}$ carbon dots, ${ }^{6}$ and boron carbon oxynitride (BCNO) phosphor materials. ${ }^{7}$ These carbon-based nanostructured materials are exciting research subjects due to their excellent mechanical strength, electrical and thermal conductivity, optical properties, low toxicity, tunable color emission, and chemical inertness. ${ }^{2,8,9}$ They offer high potential for various applications, such as drug

${ }^{a}$ Department of Physics, Faculty of Mathematics and Natural Sciences, Institut Teknologi Bandung, Jl. Ganesha 10, Bandung, 40132, Indonesia. E-mail: ferry@fi. itb.ac.id

${ }^{b}$ Research Center for Nanosciences and Nanotechnology (RCNN), Institut Teknologi Bandung, Jl. Ganesha 10, Bandung, 40132, Indonesia

$\dagger$ Electronic supplementary information (ESI) available: Detailed illustrations of equipment design for reduction of GO by microwave-assisted method under $\mathrm{N}_{2}$-atmosphere conditions, electrical conductivity data tables for rGO. Characterization results consist of ATR-FTIR, XRD, and Raman spectra, SEM, TEM, and SAED pictures of rGO reduced by hydrazine or L-AA. See DOI: 10.1039/c7ra10013b delivery systems, tissue scaffold reinforcement, catalysts, light emitting diodes (LED), panel displays, bio-imaging, DNA labeling, and energy storage materials., ${ }^{2,10-14}$

Graphene is one of the most used carbon-based nanostructured materials for energy storage, due to its high electron mobility $\left(\sim 2 \times 10^{5} \mathrm{~cm}^{2} \mathrm{~V}^{-1} \mathrm{~s}^{-1}\right)$, excellent thermal conductivity $\left(\sim 5000 \mathrm{~W} \mathrm{~m}^{-1} \mathrm{~K}^{-1}\right)$ and electrical $\left(\sim 2000 \mathrm{~S} \mathrm{~cm}^{-1}\right)$ conductivity. ${ }^{15}$ Moreover, graphene has been successfully fabricated to be used as high-speed transistor, ${ }^{16}$ radio frequency integrated circuit (IC), ${ }^{17}$ and solar cell. ${ }^{13}$ There are several methods to produce graphene, such as exfoliation (including oxidation and reduction), epitaxial growth, chemical vapor deposition (CVD), and chemical synthesis. ${ }^{18}$ Among these reported techniques, chemical exfoliation from graphite is the most promising method to synthesize graphene in large-scale production. ${ }^{5}$

Chemical exfoliation to synthesize graphene involves three steps, i.e. oxidation of graphite, exfoliation of graphite oxide into graphene oxide (GO), and subsequent reduction to form reduced graphene oxide (rGO). ${ }^{18}$ Hummer introduced the oxidation of graphite. ${ }^{19}$ This method requires concentrated acid $\left(\mathrm{H}_{2} \mathrm{SO}_{4}\right), \mathrm{NaNO}_{3}$, and a high oxidation temperature $\left(98{ }^{\circ} \mathrm{C}\right)$. Therefore, research has been done to develop more environmentally friendly techniques. Our group has reported the synthesis of rGO with a less acidic solution and faster oxidation process. ${ }^{5}$ By modifying the Marcano method and applying microwave-assisted method during the reduction of GO, higher 
electrical conductivity $\left(1180 \mathrm{~S} \mathrm{~m}^{-1}\right)$ can be achieved compared to rGO synthesis as proposed by Hummer $\left(5 \mathrm{~S} \mathrm{~m}^{-1}\right)$ or Marcano $\left(10 \mathrm{~S} \mathrm{~m}^{-1}\right) .{ }^{20}$ rGO with high electrical conductivity can be used as an electrode material. ${ }^{21}$ Moreover, composites of rGO with polymers and polymer-metal oxide combinations (including poly(3,4-ethylene dioxythiophene) (PEDOT)-rGO- $\mathrm{Co}_{3} \mathrm{O}_{4}$, polyaniline (PANI)-rGO, and $\mathrm{PANI}^{-\mathrm{TiO}}{ }_{2}-\mathrm{rGO}$ ) have been synthesized to be used as electromagnetic wave absorption materials. ${ }^{22-24}$

Hydrazine or hydrazine hydrate is a well-known reducing agent to produce rGO. However, it is known to be a toxic and highly explosive chemical, so precautions have to be taken if it is used in large quantities. ${ }^{25}$ Despite the toxicity and explosive issues of hydrazine hydrate, it is still the most useful reductant for preparing rGO. In this study, we used hydrazine hydrate to determine whether our new development method is suitable to synthesize rGO with higher conductivity properties. Thus, after establishing the new method, we used a milder and nontoxic reducing agent for comparison, i.e. L-ascorbic acid (L-AA). Several reports on the synthesis of rGO using L-AA as reducing agent have been successfully performed. ${ }^{25-28}$ However, the reduction process takes relatively long, from $30 \mathrm{~min}$ up to 48 hours reaction time. ${ }^{25,27}$ Therefore, the development of a new method to prepare rGO with a shorter reaction time needs to be done.

There are no reports on using a microwave-assisted technique under $\mathrm{N}_{2}$-atmosphere conditions in the chemical reduction of GO. The reduction of GO using microwave-assisted method has been reported previously. ${ }^{5,29-31}$ Moreover, synthesis of $\mathrm{rGO} / \mathrm{ZnO}$ and $\mathrm{rGO}-\mathrm{Ni}$ nanohybrids has been conducted using microwave-assisted methods for photocatalytic processing and non-enzymatic amperometric glucose detection, respectively. ${ }^{32,33}$ However, in these cases the technique was performed under normal atmosphere conditions. Therefore, in our study, we present reduction of GO by microwave-assisted method under $\mathrm{N}_{2}$-atmosphere conditions to improve the electrical conductivity of the resulted rGO. For comparison, the reduction of GO was also carried out under normal conditions. Thus, we were able to investigate the influence of $\mathrm{N}_{2}$-atmosphere conditions during the reduction process. Variation of irradiation times and modes were also performed to determine the optimal reduction reaction conditions. The reduction process was carried out using hydrazine hydrate as reducing agent and later on, ascorbic acid was used as an environmentally friendly reducing agent. Furthermore, we also introduce annealing under normal atmosphere to increase the electrical conductivity properties of the rGO samples.

\section{Materials and methods}

\section{Materials}

Graphite flakes, concentrated sulfuric acid $\left(\mathrm{H}_{2} \mathrm{SO}_{4}, 98 \%\right)$, potassium permanganate $\left(\mathrm{KMnO}_{4}\right)$, hydrazine hydrate $\left(\mathrm{N}_{2} \mathrm{H}_{4}\right)$, and hydrochloric acid $(\mathrm{HCl})$ were purchased from Merck, Indonesia. Phosphoric acid $\left(\mathrm{H}_{3} \mathrm{PO}_{4}\right)$, hydrogen peroxide $\left(\mathrm{H}_{2} \mathrm{O}_{2}\right)$, and ethylene glycol were obtained from Brataco Chemica, Indonesia. Deionized water and ethanol were acquired from
Smartlab, Indonesia. L-Ascorbic acid was purchased from Sigma Aldrich, Germany.

\section{Synthesis of reduced graphene oxide (rGO)}

The graphite oxide was prepared using the same method as previously reported by our group. ${ }^{5}$ Suspensions of GO were prepared by dispersing graphite oxide ( $1 \mathrm{~g}$ ) into $134 \mathrm{~mL}$ ethylene glycol (EG). Dispersing process was carried out by sonication, and subsequent exfoliation was performed by ultraturrax for $2 \mathrm{~h}$. During the stirring process, rGO was prepared by adding $1 \mathrm{~mL}$ hydrazine hydrate $\left(\mathrm{N}_{2} \mathrm{H}_{4}\right)$. The reduction process of GO was completed by introducing the sample to microwave irradiation (Panasonic Microwave $2.45 \mathrm{MHz}, 800 \mathrm{~W}$ ) at various irradiation levels (low, medium, and high). The reduction process was also performed with different irradiation times $(1,2,3,4$, and 5 minutes) under $\mathrm{N}_{2}$-flow or normal atmosphere conditions. The low mode of the microwave irradiation indicates $17 \%$ of the duty cycle, the medium mode indicates $80 \%$ of the duty cycle, and the high mode indicates $100 \%$ of the duty cycle, as shown in Fig. S1 in the ESI. $\dagger$ Afterward, the sample was filtered and subsequently washed using deionized water and alcohol. The sample was dried for $12 \mathrm{~h}$ at $60{ }^{\circ} \mathrm{C}$. Later on, rGO pellets were prepared by lab hot press equipment, but without heat treatment, and subsequently annealed at $300{ }^{\circ} \mathrm{C}$ for various annealing times $(15,30$, and 45 minutes).

Reduction of GO was also performed by using L-ascorbic acid (L-AA) as reducing agent. L-AA (8 g) was diluted in $20 \mathrm{~mL}$ of deionized water. The L-AA solution was added to suspensions of GO. Subsequently, the same reduction process was used as with hydrazine hydrate as reducing agent.

\section{Instrumental methods}

The resulting rGO powder was characterized using the following instrumental methods. Attenuated Total Reflectance-Fourier Transform Infrared Spectrometry (ATR-FTIR) measurements were carried out using Alpha FTIR Spectrometer Bruker 1176 397. X-ray diffraction (XRD) measurement was performed on a Phillips Analytical PW 1710 BASED. Conductivity properties were measured using a four-point probe, which involves four electrodes from one electrical source of an Advantest R6240A DC Voltage and Keithley multimeter. Prior to the conductivity measurements, the rGO powder was prepared as pellets using lab hot press equipment, but without heat treatment, as shown in Fig. S2 in the ESI. $\dagger$ Scanning electron microscopy (SEM) was operated at $15 \mathrm{kV}$. Transmission electron microscopy (TEM) and energy dispersive X-ray spectrometry (EDX) measurements were performed using a JEOL-JSN-6510LV. Raman spectrometry measurement was carried out using an Horiba Jobin Yvon Modular Raman spectrometer and a StellarPro Argon/Ion Laser with excitation wavelength at $514 \mathrm{~nm}$ (green) and powered at $50 \mathrm{~mW}$.

\section{Results and discussion}

rGO was prepared by reduction of graphene oxide (GO). Synthesis of GO was carried out by modified Hummer method, 
using phosphate acid instead of $\mathrm{NaNO}_{3}$. This modification is necessary due to the production of toxic gasses $\left(\mathrm{NO}_{2}\right.$ and $\left.\mathrm{N}_{2} \mathrm{O}_{4}\right)$ during the oxidation reaction and residual ions $\left(\mathrm{Na}^{+}\right.$and $\left.\mathrm{NO}_{3}{ }^{-}\right)$ that are difficult to remove after the oxidation process. ${ }^{20}$ Moreover, from the previous result reported by our group, ${ }^{5}$ modification of the Marcano method by using phosphate acid ${ }^{20}$ and microwave-assisted method, successfully resulted in higher conductivity of rGO $\left(11.80 \mathrm{~S} \mathrm{~cm}^{-1}\right)$ and decreased the production of toxic gasses during the oxidation process. ${ }^{5}$

The reduction of GO was carried out by microwave-assisted method. The change in appearance of the sample before and after the reduction process can be used as an indicator whether the reaction was completed. Fig. 1(a) and (b) show the appearance of the sample before and after reduction, respectively. The GO suspension first looked slightly brown, while after the reduction process, the sample appeared to be black, which indicates that rGO was formed. After the reduction was completed, the sample was filtered, washed and subsequently dried at $60{ }^{\circ} \mathrm{C}$ for $12 \mathrm{~h}$. The resulting rGO powder is shown in Fig. 1(c).

The reduction process was performed under normal or $\mathrm{N}_{2}$ atmosphere conditions. Prior to applying $\mathrm{N}_{2}$-atmosphere conditions, normal conditions were used to determine the influence of $\mathrm{N}_{2}$-atmosphere conditions on the reduction process. While using microwave-assisted method in normal conditions, we used various microwave irradiation levels (low, medium, and high). After 5 minutes of microwave-assisted method at various irradiation levels, it was observed that the solution of the reaction mixture evaporated rapidly when the high irradiation mode was used. Thus, by the end of the reduction process, no solution was left in the beaker glass. On the other hand, when the high irradiation mode was used under $\mathrm{N}_{2}$-flow for 5 minutes of reduction, the evaporation remained low. By flowing $\mathrm{N}_{2}$ to the reduction system, the reduction of GO by the reducing agent is more efficient, since gaseous side products will be removed from the system. Moreover, in the reduction of GO by the microwave-assisted method under normal conditions, the presence of $\mathrm{O}_{2}$ or $\mathrm{H}_{2} \mathrm{O}$ could lead to oxidation of rGO that has formed during reduction, resulting in a more ineffective process. Therefore, by applying $\mathrm{N}_{2}$-atmosphere conditions in the microwave-assisted method, the reduction of GO will be faster, more effective and efficient.

\section{Structure and morphology analysis}

Functional group characterization was accomplished by ATRFTIR spectroscopy measurements. The ATR-FTIR spectra of
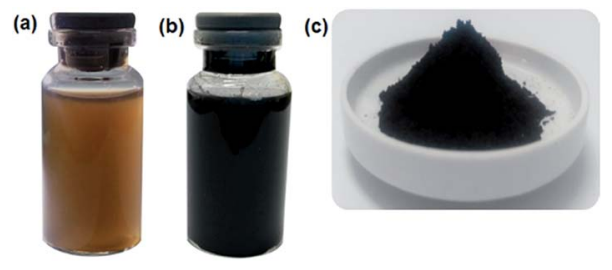

Fig. 1 Sample pictures of (a) suspension of GO, (b) completed reduction of GO using hydrazine hydrate by microwave-assisted heating, and (c) rGO powder. graphite, graphene oxide (GO), and reduced graphene oxide (rGO) are shown in Fig. 2(a). The presence of characteristic peaks at $3151,1719,1618,1221$, and $1041 \mathrm{~cm}^{-1}$ in the spectrum of the $\mathrm{GO}$ were caused by $\mathrm{O}-\mathrm{H}$ stretching, $\mathrm{C}=\mathrm{O}$ stretching, aromatic $\mathrm{C}=\mathrm{C}$ stretching, $\mathrm{O}-\mathrm{H}$ deformation, epoxy $\mathrm{C}-\mathrm{O}$ stretching, and alkoxy $\mathrm{C}-\mathrm{O}$ stretching vibration, respectively., ${ }^{5,27,34}$ These peaks do not appear in the spectrum of graphite and thus indicate the presence of a large number of oxygen-containing functional groups $(-\mathrm{COOH}$ and $\mathrm{C}=\mathrm{O}$ located at the sheet edge, $-\mathrm{OH}$ and epoxy $\mathrm{C}-\mathrm{O}$ on the basal planes of the GO sheet) that are introduced during the oxidation step..$^{35}$ After reduction by hydrazine hydrate in $\mathrm{N}_{2}$-atmosphere reaction conditions, these characteristic peaks disappeared, which indicates the removal of the oxygen-containing functional groups to a certain degree. The ATR-FTIR spectra of GO reduced at various irradiation levels are shown in Fig. S3. $\dagger$ The current peak at $1618 \mathrm{~cm}^{-1}$ in the spectra suggests that the $\mathrm{sp}^{2}$ structure of carbon atoms remained. This result is in line with the result previously reported by Su et al. ${ }^{36}$ The same results were also observed from the rGO reduced by L-AA, as shown in Fig. S4. $\dagger$

The XRD spectra of graphite, GO, and rGO are depicted in Fig. 2(b). The XRD measurements were performed to distinguish the structure changing from graphite to GO and then from GO to rGO. The XRD spectrum of the graphite is in good agreement with JCPDS 01-0640..$^{37}$ The strong and sharp peak (002) at $2 \theta=26.3^{\circ}$ is due to the highly ordered structure of the interlayer spacing of $0.33 \mathrm{~nm},{ }^{38}$ which is a characteristic peak for graphite. Modification of the graphite to GO resulted in a new peak $\left(2 \theta=10.9^{\circ}\right)$ and the characteristic peak for graphite disappeared. This result is in line with the reported results of GO prepared using the Hummer method. The diffraction peak

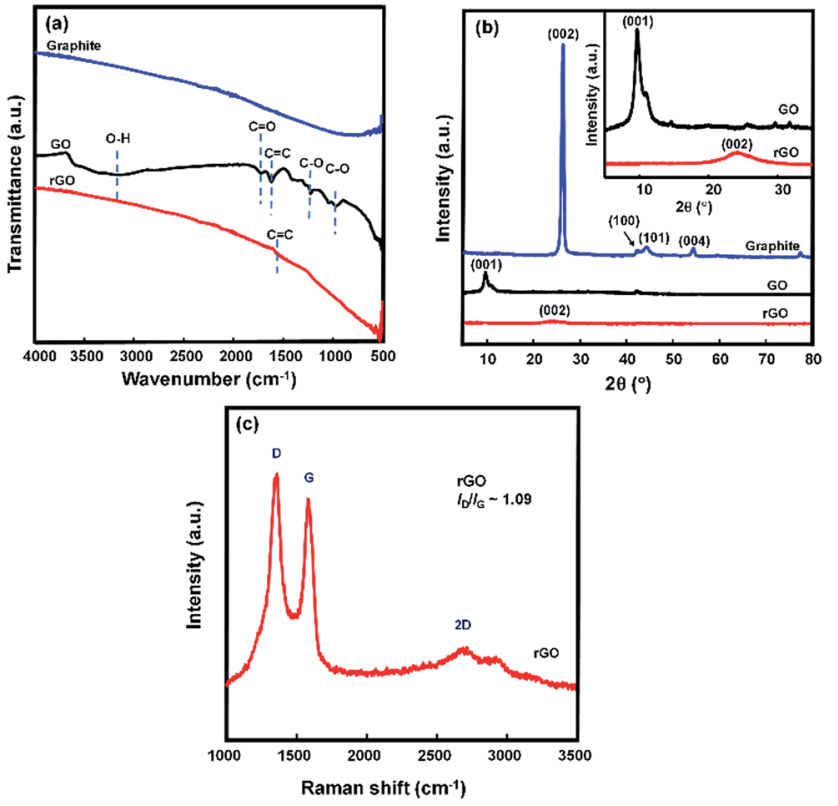

Fig. 2 (a) ATR-FTIR spectra of graphite, GO, and rGO, (b) XRD spectra of graphite, GO, and $\mathrm{rGO}$, and (c) Raman spectrum of rGO reduced using hydrazine hydrate for 3 minutes at high microwave irradiation mode under $\mathrm{N}_{2}$-atmosphere conditions. 
shifting is because of intercalating oxygen atoms during the oxidation process using $\mathrm{KMnO}_{4}$, which indicates an increase in stretching of the interlayer spacing to $0.81 \mathrm{~nm}$. The increased distance between the layers of the GO sample is due to exfoliation of the graphite layers, the addition of oxygen-containing functional groups and water molecules, ${ }^{38}$ and defects in the carbon $\mathrm{sp}^{3}$ structure of the graphite. ${ }^{39}$

The characteristic diffraction peak of GO disappeared after the reduction process. A low-intensity and broad peak was observed at $2 \theta=24.1^{\circ}$ in the XRD spectrum of the rGO, which confirms that the reduction process was successfully performed, with an interlayer spacing of $0.368 \mathrm{~nm}$. The XRD spectrum of rGO reduced by L-AA displayed the same pattern as the rGO reduced by hydrazine, as shown in Fig. S5. $\dagger$ The decrease in diffraction peak intensity compared to the peak diffraction of graphite is caused by residual oxygen-containing functional groups, which leads to a reduction in crystallinity and van der Waals interaction between layers. ${ }^{38}$

Further structure characterization was performed using Raman spectroscopy measurement. The Raman spectrum of graphene consists of three main peaks: the $\mathrm{D}$ peak at $\sim 1350 \mathrm{~cm}^{-1}$ belongs to $\mathrm{sp}^{3}$ carbon atoms from defects (in highquality graphene, the D mode is practically absent), the G peak at $\sim 1580 \mathrm{~cm}^{-1}$ corresponds to $\mathrm{sp}^{2}$ carbon atoms, and an intense $2 \mathrm{D}$ (also known as $\mathrm{G}^{\prime}$ ) peak at $\sim 2700 \mathrm{~cm}^{-1}$ represents the second-order mode of the $\mathrm{D}$ band. ${ }^{21}$ The $\mathrm{G}$ mode is correlated to the vibration of $\mathrm{sp}^{2}$ carbon atoms and the $\mathrm{D}$ mode is attributed to the conversion of $\mathrm{sp}^{2}$ carbon to $\mathrm{sp}^{3}$ carbon atoms. ${ }^{40}$ Fig. 2(c) shows the Raman spectrum of the rGO. It has two prominent peaks at 1354 and $1576 \mathrm{~cm}^{-1}$, which are attributed to the D mode and the $\mathrm{G}$ mode, respectively. The $\mathrm{D} / \mathrm{G}$ peak area ratio results for the rGO reduced by hydrazine and the rGO reduced by L-AA were 1.09 and 1.05, respectively, as shown in Fig. S6. $\dagger$ The peak intensity of the $\mathrm{D}$ and $\mathrm{G}$ band $\left(I_{\mathrm{D}} / I_{\mathrm{G}}\right)$ ratio is an estimation of the disorder level in the graphene. ${ }^{41}$ A higher $I_{\mathrm{D}} / I_{\mathrm{G}}$ ratio leads to higher defect in the graphene sheet. The $I_{\mathrm{D}} / I_{\mathrm{G}}$ ratio of $\sim 1$ of the rGO is a typical result for graphene produced by chemical exfoliation..$^{5,41,42}$

Morphology studies of rGO were performed by SEM and TEM, as shown in Fig. 3(a) and (b). Thin sheets of rGO can be observed in Fig. 3(a) and (b). In Fig. 3(b), the transparent areas represent the monolayer form of graphene and rGO. The dark areas represent a wrinkled structure that is forming a thick stacking structure of several graphene layers with an amount of oxygen-containing functional groups. The SAED pattern is depicted in Fig. 3(c), which shows rGO sheets as polynanocrystal. Similar pictures of SEM, TEM, and SAED patterns are presented in Fig. S7.†

\section{Electrical conductivity of rGO}

The electrical conductivity of the rGO was measured by fourpoint probe. For the rGO prepared by microwave-assisted method under normal atmosphere conditions, the electrical conductivity results are shown in Fig. 4(a). By increasing the irradiation level of the microwave to high, the rGO exhibited its highest electrical conductivity at $17.08 \mathrm{~S} \mathrm{~cm}^{-1}$. The higher the
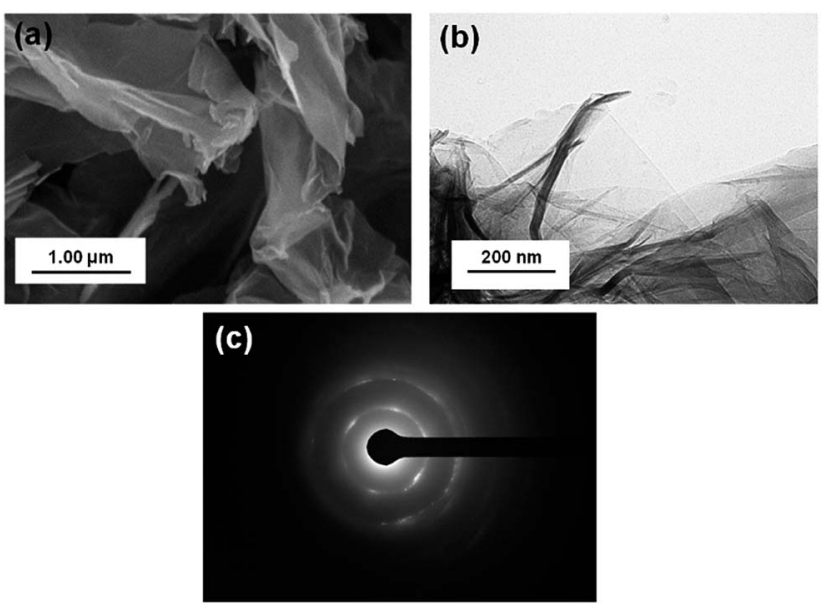

Fig. 3 Morphology pictures of (a) SEM, (b) TEM, and (c) SAED from rGO reduced by hydrazine hydrate treated with high microwave irradiation mode for 3 minutes under $\mathrm{N}_{2}$-atmosphere conditions.

irradiation level of the microwave the less reaction time was needed to produce rGO with high conductivity properties. In high irradiation mode (see Fig. 4(a)), the conductivity of the rGO increased up to $17.08 \mathrm{~S} \mathrm{~cm}^{-1}$ and then decreased to $14.64 \mathrm{~S} \mathrm{~cm}^{-1}$. Thus, by using high irradiation, optimal reduction took 4 minutes and after that, the microwave irradiation could probably disrupt the $\mathrm{sp}^{2}$ structure of carbon atoms, which resulted in a decrease of the electrical conductivity.

The electrical conductivity study of the rGO prepared by microwave-assisted method in high irradiation mode under $\mathrm{N}_{2}$ atmosphere conditions is shown in Fig. 4(b). By applying $\mathrm{N}_{2}$ atmosphere conditions in the reduction of GO, the rGO resulted from the irradiation for 3 minutes showed its highest conductivity at $18.10 \mathrm{~S} \mathrm{~cm}^{-1}$. This conductivity result is higher compared to previously reported results. ${ }^{5}$ Increasing the irradiation time to more than 3 minutes resulted in a decrease of the conductivity properties of the rGO. This indicates the presence of more defects in the $\mathrm{sp}^{2}$ structure of carbon atoms. This result is consistent with the conductivity results of rGO prepared by microwave-assisted method under normal atmosphere conditions.
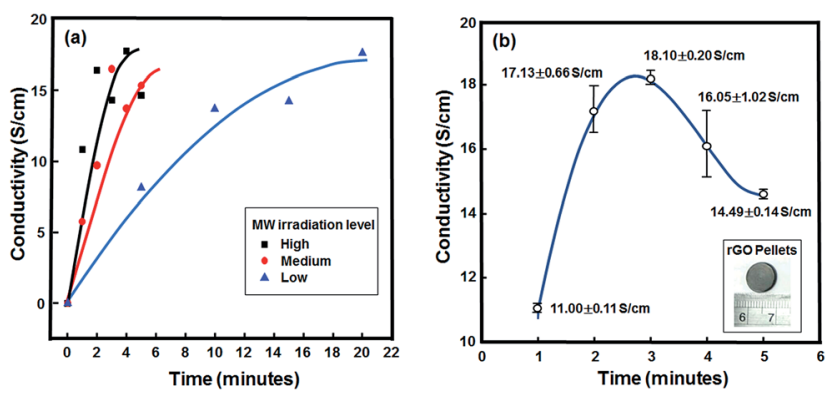

Fig. 4 Electrical conductivity results of (a) influence of microwave irradiation levels (under normal atmosphere conditions), and (b) variation of irradiation times under $\mathrm{N}_{2}$ atmosphere conditions. 
From the above results, it can be seen that rGO was successfully prepared by microwave-assisted method under $\mathrm{N}_{2}$ atmosphere conditions using hydrazine hydrate as reducing agent. A conductivity of $18.10 \mathrm{~S} \mathrm{~cm}^{-1}$ was achieved from the rGO prepared by 3 minutes of microwave irradiation in high mode using hydrazine hydrate as reducing agent. With L-AA as reducing agent, the same reducing conditions were used as with hydrazine hydrate.

The annealing process was conducted to further improve the electrical conductivity of the rGO prepared by microwaveassisted method under $\mathrm{N}_{2}$-atmosphere conditions. Before the annealing process, the rGO pellets were made by lab hot press equipment, but without heat treatment. The electrical conductivity results of the rGO reduced by hydrazine hydrate and by LAA are shown in Fig. 5. Various annealing times were applied to determine the effect of annealing on the electrical conductivity properties. Without annealing treatment, the electrical conductivity of the rGO reduced by hydrazine hydrate was $18.10 \mathrm{~S} \mathrm{~cm}^{-1}$. By treating the rGO pellets with annealing at $300{ }^{\circ} \mathrm{C}$ for $30 \mathrm{~min}$, the electrical conductivity increased up to $23.26 \mathrm{~S} \mathrm{~cm}^{-1}$. However, increasing the annealing time to $45 \mathrm{~min}$ decreased the electrical conductivity to $22.36 \mathrm{~S} \mathrm{~cm}^{-1}$. A similar pattern was observed for the rGO reduced by 3 minutes of irradiation in high mode using $\mathrm{L}_{-\mathrm{AA}}$ as the reducing agent (rGOLAA). The rGO-LAA without annealing treatment had an electrical conductivity of $12.02 \mathrm{~S} \mathrm{~cm}^{-1}$. The electrical conductivity of the rGO-LAA gradually increased up to $16.19 \mathrm{~S} \mathrm{~cm}^{-1}$ when the annealing treatment was applied for $30 \mathrm{~min}$ and decreased to $15.80 \mathrm{~S} \mathrm{~cm}^{-1}$ when annealing was applied for $45 \mathrm{~min}$.

Although the electrical conductivity of the rGO reduced by L-AA was lower than that of the rGO reduced by hydrazine hydrate, the conductivity result was still higher than the reported results. ${ }^{25,27}$ The lower conductivity results are due to the difference in reduction degree of the rGO. From a comparison of the EDX results of the rGO reduced by hydrazine hydrate and those of the rGO reduced by L-AA (as shown in Fig. 6), the oxygen-containing groups of the rGO reduced by hydrazine hydrate were removed better than in the rGO reduced by L-AA. Therefore, the use of L-AA

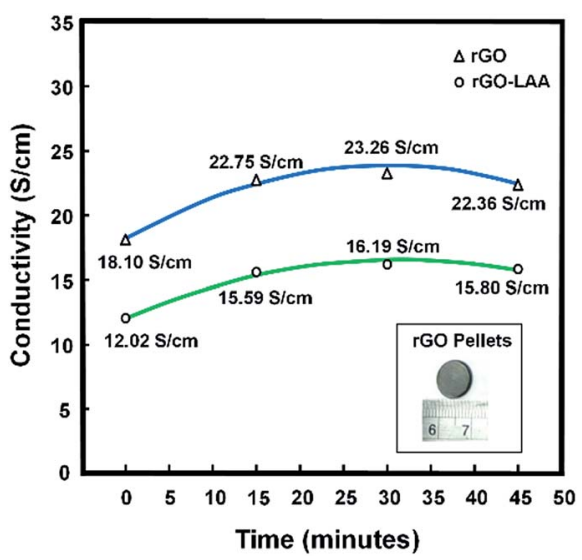

Fig. 5 Influence of annealing time on electrical conductivity of rGO reduced by hydrazine hydrate and $\mathrm{rGO}$ reduced by L-AA.
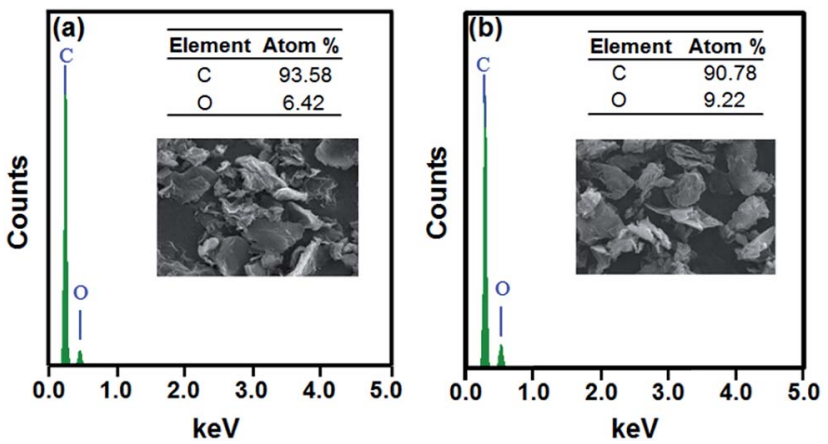

Fig. 6 EDX results of (a) rGO reduced by hydrazine hydrate and (b) rGO reduced by L-AA.

as reducing agent with microwave-assisted treatment under $\mathrm{N}_{2}$ atmosphere conditions still needs to be optimized.

The electrical conductivity results show that annealing successfully improved the conductivity properties of the rGO. This happens due to the reduction process that occurs during the annealing process, which leads to more $\mathrm{sp}^{2}$ structures of carbon atoms and results in more $\pi-\pi$ electron conjugations, thus leading to an increase in electrical conductivity. However, increasing the annealing time leads not only to more oxygen removal from rGO but it also disrupts the $\mathrm{sp}^{2}$ structures of the rGO. Therefore, the electrical conductivity decreased after 45 minutes of annealing.

Fig. 7 displays the possible reaction mechanism of the reduction of graphene oxide (GO) using (a) hydrazine hydrate, (b) L-ascorbic acid (L-AA), and (c) annealing. In the reduction of GO using hydrazine hydrate as a reducing agent, the reaction follows the blue path Fig. $7\left(a_{1}\right)$ and $\left(a_{2}\right)$. In Fig. $7\left(a_{1}\right)$, the reduction is based on the Wolff-Kishner reduction mechanism, which starts with a nucleophilic attack of hydrazine on the carbon atom of the carbonyl functional group. The resulted products from this reduction are alkene and $\mathrm{N}_{2}$ gas. By using hydrazine hydrate, the reduction can also be performed on the epoxide ring that follows the path in Fig. $7\left(\mathrm{a}_{2}\right)$. This reduction mechanism is based on the research previously reported by Gao et $a .^{\mathbf{4 3}}$ The reduction of epoxide ring (de-epoxidation) starts with the hydrazine attack on the carbon atom of the backside of the epoxide rings to form the hydrazino alcohol. This is followed by hydrogen transfer from the hydrazino group to an alcohol group, which results in the formation of $\mathrm{C}=\mathrm{C}$, water, and cis-diazene (cis- $\mathrm{N}_{2} \mathrm{H}_{2}$ ).

The reduction mechanism of GO using L-AA as a reducing agent takes the green path in Fig. $7\left(b_{1}\right)$ and $\left(b_{2}\right)$, which represents the reduction of the epoxide ring and the hydroxyl functional group, respectively. These possible reaction mechanisms are based on the previously reported result of $\mathrm{Xu}$ et al. ${ }^{27} \mathrm{In}$ Fig. $7\left(b_{1}\right)$, the nucleophilic attack of oxygen from the hydroxyl group of the L-AA to the carbon in the vinyl alcohol can be seen. Subsequently, thermal elimination will restore the $\mathrm{C}=\mathrm{C}$ bond and the resulting byproducts are dehydroascorbic acid and a water molecule. A similar reduction mechanism also occurs on the epoxide ring in the graphene sheet. The nucleophilic attack by the hydroxyl group of the L-AA on the carbon atoms of 


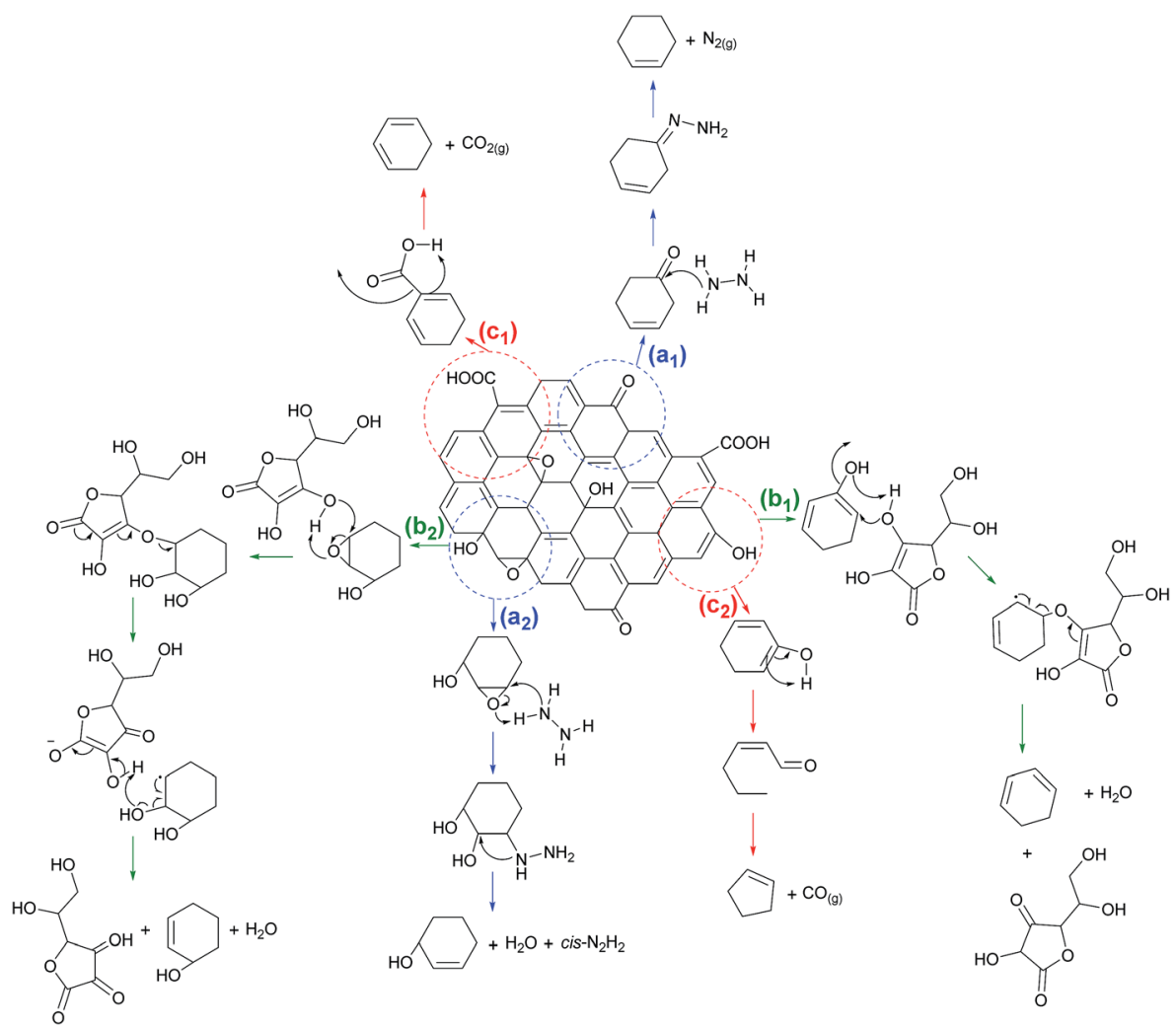

Fig. 7 Possible reduction GO reaction mechanism by (a) hydrazine hydrate, (b) L-AA, or (c) thermal during the annealing process.

the epoxide ring resulted in $\mathrm{C}=\mathrm{C}$ and byproducts, as shown in Fig. $7\left(b_{2}\right)$.

The reduction process can be carried out using a heating treatment, which is shown by the red path in Fig. $7\left(c_{1}\right)$ and $\left(c_{2}\right)$. The reduction process occurs during the annealing process that is based on the previously reported mechanism of thermal decarboxylation and decarbonylation. ${ }^{43}$ The reduction process via decarboxylation is shown in Fig. $7\left(c_{1}\right)$. The decarboxylation mechanism occurs slowly at temperatures of $100-150{ }^{\circ} \mathrm{C} .{ }^{43}$ Since the annealing process was done at $300{ }^{\circ} \mathrm{C}$, the rGO product indeed underwent thermal decarboxylation. The byproduct from this thermal decarboxylation is $\mathrm{CO}_{2}$ gas. Another possible reduction mechanism is via decarbonylation, which is shown in Fig. $7\left(c_{2}\right)$. This thermal reduction occurs on the hydroxyl group attached to the aromatic ring, which results in the formation of aldehyde and subsequent heating leads to decarbonylation of the aldehyde to form CO gas. This decarbonylation occurs easier for hydroxyl groups attached to the interior of an aromatic domain compared to those on the edges of the aromatic domain. This thermal decarbonylation produces a defect in the graphene sheet, which is due to the formation of a five-membered ring structure. According to the simulation calculation of the Gibbs free-energy barrier by Gao et al., ${ }^{\mathbf{4 3}}$ thermal decarbonylation occurs at a temperature higher than $700{ }^{\circ} \mathrm{C}$. In this research, we did the annealing process at $300{ }^{\circ} \mathrm{C}$ thus decarbonylation may not have occurred. However, when the annealing temperature at $300{ }^{\circ} \mathrm{C}$ was extended, decarbonylation may have occurred since we observed a decrease in the electrical conductivity of the rGO.
From the reaction mechanism, extensive heating can lead to disruption of the hexagonal $\mathrm{sp}^{2}$ carbon structure and lead to form the five-membered ring structure that consequently causes the defect in graphene sheets.

\section{Conclusions}

Synthesis of rGO prepared by microwave-assisted method under normal and $\mathrm{N}_{2}$-atmosphere conditions was successfully performed. The structures and morphologies of the rGO synthesized by hydrazine hydrate and L-AA were confirmed by ATR-FTIR, XRD, Raman, SEM, TEM, and EDX results. Introducing microwaveassisted method in high mode under $\mathrm{N}_{2}$-atmosphere conditions resulted in a facile synthesis of rGO. The rGO prepared using this newly developed method only needed 3 minutes to complete the reduction process. The highest conductivity of $18.10 \mathrm{~S} \mathrm{~cm}^{-1}$ was achieved for the rGO prepared by this new method using hydrazine hydrate as reducing agent. Further annealing at $300{ }^{\circ} \mathrm{C}$ for 30 minutes, increased electrical conductivity up to $23.26 \mathrm{~S} \mathrm{~cm}^{-1}$. By using this new method in the synthesis of rGO with L-AA as reducing agent, an electrical conductivity of $12.02 \mathrm{~S} \mathrm{~cm}^{-1}$ was observed, which increased up to $16.19 \mathrm{~S} \mathrm{~cm}^{-1}$ after annealing. The combination of this new method and the further annealing process of the rGO resulted in rGO with high electrical conductivity.

\section{Conflicts of interest}

There are no conflicts to declare. 


\section{Acknowledgements}

This work was supported by Program Penelitian, Pengabdian kepada Masyarakat dan Inovasi (P3MI), Institut Teknologi Bandung (ITB). We gratefully acknowledge Mr. Dadang Suhendra for his assistance in the modification of the microwave equipment for achieving $\mathrm{N}_{2}$-atmosphere conditions. We would like to thank Dr. Arramel and Ms. Tan Wei Ling (National University of Singapore) for their kind assistance in Raman measurements. U. Hikmah acknowledges Lembaga Pengelola Dana Pendidikan (LPDP), Ministry of Finance of Indonesia for their scholarship. E. Stavila thanks the Insentif Postdoc Program, ITB, for their financial support.

\section{References}

1 G. S. Patrick and M. S. R. Rao, J. Phys. D: Appl. Phys., 2010, 43, 370301.

2 C. Cha, S. R. Shin, N. Annabi, M. R. Dokmeci and A. Khademhosseini, ACS Nano, 2013, 7, 2891-2897.

3 B.-T. Zhang, X. Zheng, H.-F. Li and J.-M. Lin, Anal. Chim. Acta, 2013, 784, 1-17.

4 F. A. Permatasari, A. H. Aimon, F. Iskandar, T. Ogi and K. Okuyama, Sci. Rep., 2016, 6, 21042.

5 H. Miftahul, A. F. Hafizh, F. Rohman, H. A. Akfiny and I. Ferry, Mater. Res. Express, 2017, 4, 064001.

6 T. Ogi, K. Aishima, F. A. Permatasari, F. Iskandar, E. Tanabe and K. Okuyama, New J. Chem., 2016, 40, 5555-5561.

7 B. Nuryadin, F. A. Permatasari, A. Y. Nuryantini, I. D. Faryuni, M. Abdullah and F. Iskandar, RSC Adv., 2017, 7, 4161-4166.

8 D. Qu, M. Zheng, L. Zhang, H. Zhao, Z. Xie, X. Jing, R. E. Haddad, H. Fan and Z. Sun, Sci. Rep., 2014, 4, 5294.

9 L. Tang, R. Ji, X. Li, K. S. Teng and S. P. Lau, J. Mater. Chem. C, 2013, 1, 4908-4915.

10 X. Liu, S. Ye, Y. Qiao, G. Dong, Q. Zhang and J. Qiu, Chem. Commun., 2009, 4073-4075, DOI: 10.1039/b904567h.

11 X. Zhang, L. Li, Z. Lu, J. Lin, X. Xu, Y. Ma, X. Yang, F. Meng, J. Zhao and C. Tang, J. Am. Ceram. Soc., 2014, 97, 246-250.

12 T. Kuila, A. K. Mishra, P. Khanra, N. H. Kim and J. H. Lee, Nanoscale, 2013, 5, 52-71.

13 X. Li, W. Chen, S. Zhang, Z. Wu, P. Wang, Z. Xu, H. Chen, W. Yin, H. Zhong and S. Lin, Nano Energy, 2015, 16, 310-319.

14 X. Chen, Y. Huang, K. Zhang, X. Feng and M. Wang, Chem. Eng. J., 2017, 330, 470-479.

15 S. Park and R. S. Ruoff, Nat. Nanotechnol., 2009, 4, 217-224. 16 Y.-M. Lin, K. A. Jenkins, A. Valdes-Garcia, J. P. Small, D. B. Farmer and P. Avouris, Nano Lett., 2009, 9, 422-426.

17 S.-J. Han, A. V. Garcia, S. Oida, K. A. Jenkins and W. Haensch, Nat. Commun., 2014, 5, 3086.

18 N. O. Weiss, H. Zhou, L. Liao, Y. Liu, S. Jiang, Y. Huang and X. Duan, Adv. Mater., 2012, 24, 5782-5825.

19 W. S. Hummers and R. E. Offeman, J. Am. Chem. Soc., 1958, 80, 1339.
20 D. C. Marcano, D. V. Kosynkin, J. M. Berlin, A. Sinitskii, Z. Sun, A. Slesarev, L. B. Alemany, W. Lu and J. M. Tour, ACS Nano, 2010, 4, 4806-4814.

21 D. Voiry, J. Yang, J. Kupferberg, R. Fullon, C. Lee, H. Y. Jeong, H. S. Shin and M. Chhowalla, Science, 2016, 353, 1413.

22 P. Liu, Y. Huang, J. Yan and Y. Zhao, J. Mater. Chem. C, 2016, 4, 6362-6370.

23 P.-B. Liu, Y. Huang and X. Sun, ACS Appl. Mater. Interfaces, 2013, 5, 12355-12360.

24 J. Yan, Y. Huang, C. Wei, N. Zhang and P. Liu, Composites, Part A, 2017, 99, 121-128.

25 J. Zhang, H. Yang, G. Shen, P. Cheng, J. Zhang and S. Guo, Chem. Commun., 2010, 46, 1112-1114.

26 X. Zhu, Q. Liu, X. Zhu and Y. Liang, Int. J. Electrochem. Sci., 2012, 7, 5172-5184.

27 C. Xu, X. Shi, A. Ji, L. Shi, C. Zhou and Y. Cui, PLoS One, 2015, 10, e0144842.

28 M. J. Fernández-Merino, L. Guardia, J. I. Paredes, S. VillarRodil, P. Solís-Fernández, A. Martínez-Alonso and J. M. D. Tascón, J. Phys. Chem. C, 2010, 114, 6426-6432.

29 Y. Zhu, S. Murali, M. D. Stoller, A. Velamakanni, R. D. Piner and R. S. Ruoff, Carbon, 2010, 48, 2118-2122.

30 W. Chen, L. Yan and P. R. Bangal, Carbon, 2010, 48, 11461152.

31 H. Hu, Z. Zhao, Q. Zhou, Y. Gogotsi and J. Qiu, Carbon, 2012, 50, 3267-3273.

32 Y. Liu, Y. Hu, M. Zhou, H. Qian and X. Hu, Appl. Catal., B, 2012, 125, 425-431.

33 Z. Wang, Y. Hu, W. Yang, M. Zhou and X. Hu, Sensors, 2012, 12, 4860-4869.

34 C. Zhu, S. Guo, Y. Fang and S. Dong, ACS Nano, 2010, 4, 2429-2437.

35 S. Stankovich, D. A. Dikin, G. H. B. Dommett, K. M. Kohlhaas, E. J. Zimney, E. A. Stach, R. D. Piner, S. T. Nguyen and R. S. Ruoff, Nature, 2006, 442, 282-286.

36 X. Su, G. Wang, W. Li, J. Bai and H. Wang, Adv. Powder Technol., 2013, 24, 317-323.

37 L. Osmieri, A. H. A. Monteverde Videla and S. Specchia, J. Power Sources, 2015, 278, 296-307.

38 X. Leng, R.-t. Liu, J.-p. Zou, X. Xiong and H.-w. He, J. Cent. South Univ., 2016, 23, 1823-1830.

39 M. Aslam, M. A. Kalyar and Z. A. Raza, Mater. Res. Express, 2016, 3, 105036.

40 Z. Li, Y. Yao, Z. Lin, K.-S. Moon, W. Lin and C. Wong, J. Mater. Chem., 2010, 20, 4781-4783.

41 S. Abdolhosseinzadeh, H. Asgharzadeh and H. Seop Kim, Sci. Rep., 2015, 5, 10160.

42 Y. Zhou, Q. Bao, L. A. L. Tang, Y. Zhong and K. P. Loh, Chem. Mater., 2009, 21, 2950-2956.

43 X. Gao, J. Jang and S. Nagase, J. Phys. Chem. C, 2010, 114, 832-842. 